\title{
Combined Cardiac and Respiratory Motion Compensation for Atrial Fibrillation Ablation Procedures
}

\author{
Alexander Brost ${ }^{1}$, Wen Wu ${ }^{2}$, Martin Koch ${ }^{1}$, Andreas Wimmer ${ }^{1}$, \\ Terrence Chen ${ }^{2}$, Rui Liao ${ }^{2}$, Joachim Hornegger ${ }^{1}$, and Norbert Strobel ${ }^{3}$ \\ 1 Pattern Recognition Lab, Friedrich-Alexander-University \\ Erlangen-Nuremberg, Erlangen, Germany \\ Alexander.Brost@informatik. uni-erlangen.de \\ 2 Siemens Corporate Research, Princeton, NJ, USA \\ 3 Siemens AG, Forchheim, Germany
}

\begin{abstract}
Catheter ablation of atrial fibrillation has become an accepted treatment option if a patient no longer responds to or tolerates drug therapy. A main goal is the electrical isolation of the pulmonary veins attached to the left atrium. Catheter ablation may be performed under fluoroscopic image guidance. Due to the rather low soft-tissue contrast of X-ray imaging, the heart is not visible in these images. To overcome this problem, overlay images from pre-operative 3 -D volumetric data can be used to add anatomical detail. Unfortunately, this overlay is compromised by respiratory and cardiac motion. In the past, two methods have been proposed to perform motion compensation. The first approach involves tracking of a circumferential mapping catheter placed at an ostium of a pulmonary vein. The second method relies on a motion estimate obtained by localizing an electrode of the coronary sinus (CS) catheter. We propose a new motion compensation scheme which combines these two methods. The effectiveness of the proposed method is verified using 19 real clinical data sets. The motion in the fluoroscopic images was estimated with an overall average error of $0.55 \mathrm{~mm}$ by tracking the circumferential mapping catheter. By applying an algorithm involving both the CS catheter and the circumferential mapping catheter, we were able to detect motion of the mapping catheter from one pulmonary vein to another with a false positive rate of $5.8 \%$.
\end{abstract}

\section{Introduction}

Atrial fibrillation (AFib) is widely recognized as a leading cause of stroke 11. An increasingly popular treatment option for AFib is catheter ablation during which the pulmonary veins attached to the left atrium (LA) are electrically isolated [2]. These procedures are performed in electrophysiology (EP) labs often equipped with modern $\mathrm{C}$-arm X-ray systems providing 3-D imaging of the heart [3. The use of fluoroscopic overlay images, perspectively rendered from 3-D data sets, makes it possible to augment live 2-D X-ray projections with soft-tissue information 4]. Unfortunately, catheter navigation using augmented fluoroscopy may be

G. Fichtinger, A. Martel, and T. Peters (Eds.): MICCAI 2011, Part I, LNCS 6891, pp. 540-547, 2011.

(C) Springer-Verlag Berlin Heidelberg 2011 


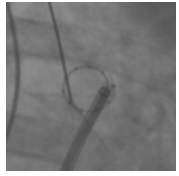

(a)

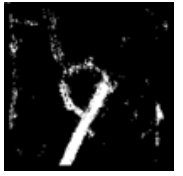

(b)

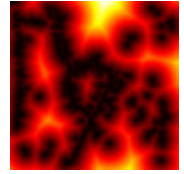

(c)

Fig. 1. (a) Cropped image for catheter segmentation. The position of the region is determined by the previous tracking result. (b) Segmented catheter using our boosted classifier approach. (c) The L2-distance transform of the segmentation after skeletonization is used as cost function for our combined registration-tracking approach.

compromised by cardiac and respiratory motion. The first approach to overcome this problem, by providing a motion compensated fluoroscopic overlay image, has been proposed in [5. It involved tracking of a commonly used circumferential mapping (CFM) catheter firmly positioned at the ostium of the pulmonary vein in simultaneous biplane images. Drawbacks of this method are simultaneous biplane imaging and a need to detect when the CFM catheter has been moved from one PV to another. Another method for respiratory motion compensation has been introduced that tracks a catheter placed in the coronary sinus (CS) vein [6]. Its drawback is that the CS catheter is far away from the site of ablation. As a consequence, it is not obvious that the motion derived from the CS catheter can be directly applied to compensation of both cardiac and respiratory motion. For example, relying on the CS catheter for motion compensation, we may encounter two problems. First, the correlation between the observed CS catheter motion and the actual motion required for dynamic overlay may be low. Second, the catheter in the coronary sinus may not always be visible due to collimation to reduce X-ray dose. Motion compensation using the CS catheter in 6] focused on 2-D motion compensation. Since simultaneous biplane fluoroscopy is hardly used in clinical practice, we also restrict ourselves to 2-D motion compensation as in 76]. We present a novel method that combines the best ideas of the previous methods. The CS catheter is used as a point of reference to detect when the circumferential mapping catheter has been moved from one PV to another, while the motion estimate for adapting the fluoroscopic overlay is derived by localizing the CFM catheter. To obtain a good anchor point along the CS catheter, we decided to introduce a virtual electrode (VE). It is placed on the CS catheter more proximal than any other electrode. Below, we briefly explain our new motion compensation method first. Then we turn to our evaluation and the results. Afterwards, we discuss our results and draw conclusions from this work.

\section{Motion Compensation}

Our motion compensation approach involves tracking of the CFM catheter as well as tracking of a VE placed on the CS catheter. The absolute distance between the center of the CFM catheter and the VE is used to identify whether the CFM has been moved from one PV to another. 


\subsection{Circumferential Mapping Catheter Tracking}

The circumferential mapping catheter is initially extracted in the 2-D X-ray image by manual interaction to get an accurate catheter model. The manual interaction is used only for the first frame of a sequence. The catheter is segmented on a region of interest, see Fig. 1(a). The position of this region is determined by the center of the tracked model from the previous frame. Haar-like features and a cascade of boosted classifiers 8 are used for segmentation. We use classification and regression trees as weak classifiers [9] which are composed to a strong classifier by the AdaBoost algorithm [10. Our tracking approach follows the method proposed in [7. A L2-distance map $\boldsymbol{I}_{\mathrm{DT}}$ [1] is calculated from the skeleton of the segmentation, see Fig. 1(c). One pixel $\boldsymbol{p}$ is accessed by $\boldsymbol{I}_{\mathrm{DT}}(\boldsymbol{p})$. Using a 2-D/2-D registration, the result is a 2-D pixel offset $t \in \mathbb{R}^{2}$. The average distance between model and segmentation derived from the image is considered as the cost value. The optimal translation $\hat{\boldsymbol{t}}$ is found by minimizing

$$
\hat{\boldsymbol{t}}=\arg \min _{\boldsymbol{t}} \sum_{i} \boldsymbol{I}_{\mathrm{DT}}\left(\boldsymbol{q}_{i}+\boldsymbol{t}\right)
$$

with $\boldsymbol{q}_{i}$ denoting a point of the catheter model. The estimated 2-D translation $\hat{t}$ is then considered as candidate for motion compensation.

\subsection{Coronary Sinus Catheter Tracking}

The coronary sinus catheter is modeled by a set of electrodes, starting from the tip of the catheter going through each individual electrode including the most proximal electrode (MPE), to the virtual electrode, see Fig. 2 (a). The VE is a reference point set by clicking on an arbitrary position along the catheter sheath that has to be more proximal than the real MPE. Similar to the circumferential mapping catheter, manual interaction is used to generate the initial CS catheter model in the first frame in a fluoroscopic sequence. For the remaining frames, all the electrodes are tracked using the approach proposed in [12. Localization of the $\mathrm{VE}$ is performed in a two-stage process. In the first stage, we robustly track all the real electrodes. In the second stage, the VE is inferred from the MPE along the CS catheter using a robust tracking framework combined with a geodesic constraint. We rely on learning-based detectors to generate hypotheses of possible models. The classifiers use Haar-like features. Each classifier is a Probabilistic Boosting Tree [13. To track the VE, specially-designed hypotheses generated by the learning-based catheter body detector are fused. Robust hypothesis matching through a Bayesian framework is then used to select the best hypothesis for each frame. Given the MPE robustly localized in the first stage, the hypotheses for tracking VE are generated automatically by a novel scheme in the second stage. The set of hypotheses is generated by parametrically manipulating the VE model based on the MPE location. A seed hypothesis $\overline{\boldsymbol{v}}_{t}$ for time step $t \in \mathbb{N}$ is generated by translating $\hat{\boldsymbol{v}}_{t-1}$, a set of 2-D image points in homogeneous coordinates, to the MPE. Then we apply a set of affine transformation to generate tracking hypotheses as 


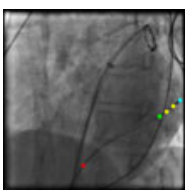

(a)

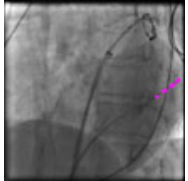

(b)

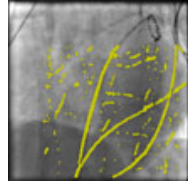

(c)

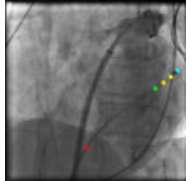

(d)

Fig. 2. CS catheter detection and VE tracking. (a) Fluoroscopic image with user inputs (electrodes and VE). (b) Automatically detected electrode positions after non-max suppression, without decision which electrode is the tip and which electrodes belong to the body. (c) Detected catheter body points. (d) Tracked electrodes and VE.

$$
\boldsymbol{v}_{t}=\left(\begin{array}{cc}
\boldsymbol{R} & \boldsymbol{t} \\
0^{T} & 1
\end{array}\right) \cdot \overline{\boldsymbol{v}}_{t}
$$

with $\boldsymbol{R} \in \mathbb{R}^{2 \times 2}$ and $\boldsymbol{t} \in \mathbb{R}^{2}$ being the parameter of an affine transformation. The MPE is used as transformation center. This strategy is efficient in generating a near-complete pool of tracking hypotheses. The overall goal for evaluating a VE tracking hypothesis is to maximize the posterior probability

$$
\hat{\boldsymbol{v}}_{t}=\arg \max _{\boldsymbol{v}_{t}} P\left(\boldsymbol{v}_{t} \mid \boldsymbol{I}_{0, \ldots, t}\right)
$$

with the image observation $\boldsymbol{I}_{0, \ldots, t}$ from 0 to $t$-th frame. The tracking is summarized in Fig. 2 ,

\subsection{Displacement Detection}

Motion compensation was performed by tracking the circumferential mapping (CFM) catheter. We settled on the CFM approach, because our data did not reveal a sufficiently strong correlation between the motion at the CS catheter and the PV ostium. To explain our findings, let us first recall that the CS catheter, placed in the coronary sinus vein, lies between the left atrium and the left ventricle. As a result, its motion may be highly influenced by the motion of the left ventricle in addition to breathing. The motion of the circumferential mapping catheter, on the other hand, is more constrained because the left atrium is connected to the lungs via the pulmonary veins. Although we decided against using the CS for motion estimation directly, we found it very useful as an anchor, i.e., to detect if the CFM catheter was moved from one PV ostium to the next. To this end, we assumed that the absolute distance between CS catheter and CFM catheter remains sufficiently stable to classify whether the CFM catheter has been moved away from a PV ostium. To achieve a reliable and robust motion compensation, we track both catheters at the same time and compare the absolute 2-D distance between the virtual electrode and the loop's center of the circumferential mapping catheter between two consecutive frames. If the distance 
Table 1. Displacement detection using the absolute difference between the CFM catheter and the VE on the CS catheter. False positive (FP) is the percentage of wrongly detected motion and false negative (FN) of undetected motion.

\begin{tabular}{|c||c|c|c|c|c|c|c|c|c|}
\hline \multicolumn{7}{|c|}{ Displacement Detection } \\
\hline \hline \multirow{2}{*}{} & $2 \%$ & $5 \%$ & $6 \%$ & $7 \%$ & $10 \%$ & $15 \%$ & $20 \%$ \\
\hline \multirow{2}{*}{$\mathrm{VE}$} & $F P$ & $22.7 \%$ & $5.8 \%$ & $4.2 \%$ & $2.7 \%$ & $0.5 \%$ & $0.0 \%$ & $0.0 \%$ \\
\cline { 2 - 9 } & $F N$ & $0.0 \%$ & $0.0 \%$ & $14.3 \%$ & $57.1 \%$ & $42.9 \%$ & $57.1 \%$ & $85.7 \%$ \\
\hline
\end{tabular}

changes by more than a certain percentage, we assume that the CFM catheter has been moved from one pulmonary vein to another. In this case, no motion compensation is applied to the fluoroscopic images until another stable CFM position has been reached. Catheter tracking is still performed. As soon as the absolute distance becomes stable again, i.e., the distance change is $\leq 5 \%$, the motion of the tracked CFM catheter is again applied to the fluoroscopic overlay.

\section{Evaluation and Results}

Our methods were evaluated on 14 clinical data sets from two different hospitals and from 10 different patients using leave-one-out validation 1 . During three of these sequences, a 10-electrode CS catheter was used. In the remaining data sets, 4 -electrode catheters were chosen. The images were either $512 \times 512$ pixels or $1024 \times 1024$ pixels. The pixel size varied between $0.173 \mathrm{~mm}$ and $0.345 \mathrm{~mm}$. Image acquisition was performed without using ECG-triggered fluoroscopy. Hence, both respiratory and cardiac motion were present. At first, we evaluated the accuracy of the tracking methods. The localization error of the detected circumferential mapping catheter was calculated by considering the 2-D Euclidean distance to a gold-standard segmentation. To this end, the L2-distance transform of the goldstandard segmentation was calculated. The 2-D Euclidean distance was then obtained as the average over all values of the distance transform when evaluating it at the 2-D positions of the detected CFM. The gold-standard segmentation was manually generated for each frame in every sequence. The segmentation was supervised by an electrophysiologist. The localization accuracy of the coronary sinus catheter and the virtual electrode was calculated as the 2-D Euclidean distance to a gold-standard segmentation of the whole CS catheter. As before, the L2-distance transform was used. The results are given in Fig. 3(a). The CFM localization yielded an average 2-D error of $0.55 \mathrm{~mm}$, which includes the inherent model error. The detection of the MPE on the CS catheter yielded an average 2$\mathrm{D}$ error of $0.52 \mathrm{~mm}$. The VE detection yielded an average 2-D error of $0.49 \mathrm{~mm}$. Further, we compared the motion calculated from the catheter detection methods to the motion observed at the PV ostia. This motion was obtained by using a gold-standard segmentation of the circumferential mapping catheter. The center of the 2-D catheter model was used to calculate the underlying motion of the

${ }^{1}$ Data is available from the authors on request for non-commercial research purposes. 


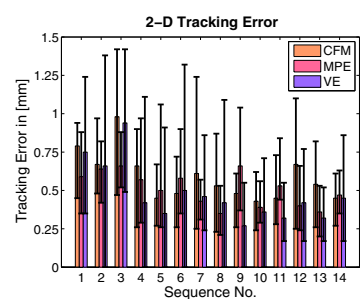

(a)

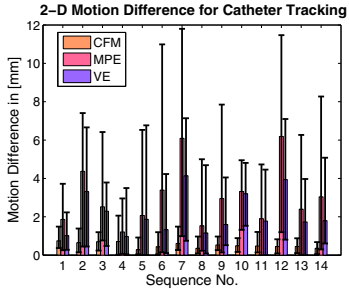

(b)

Fig. 3. (a) Accuracy for the catheter tracking methods. Tracking of the circumferential mapping (CFM) catheter, the most proximal electrode (MPE) on the CS catheter, and the virtual electrode (VE) on the CS catheter. (b) Difference between the observed motion by the circumferential mapping catheter and the catheter tracking methods.

PV between successive frames. The comparison is given in Fig. 3(b) The motion obtained by CFM catheter detection differs on average by about $0.48 \mathrm{~mm}$ from the real motion, whereas the motion from the proximal CS electrode had a mean error of about $2.61 \mathrm{~mm}$. Using the virtual electrode, we could reduce the mean error from $2.61 \mathrm{~mm}$ to $1.68 \mathrm{~mm}$. The maximum difference between the true and the estimated motion using the CFM catheter was $2.06 \mathrm{~mm}$. The MPE was off by up to $11.80 \mathrm{~mm}$ and the VE by up to $7.14 \mathrm{~mm}$, see Fig. 3(b). The 14 fluoroscopic sequences used for evaluating the tracking performance had the CFM catheter firmly placed at a single pulmonary vein, i.e., the CFM catheter was not moved from one PV to the next. To evaluate our displacement detection method, five further sequences were added to our data set. To detect CFM catheter displacement, we introduced a displacement threshold. The displacement threshold is a percentage of the distance between $\mathrm{VE}$ and the center of the loop representing the CFM catheter. Results for different displacement thresholds are given in Tab. 1. The best result for displacement detection was found for an allowed change of the distance between $5 \%$ and $10 \%$. A change in the absolute distance of $5 \%$ turned out to be the best threshold for detecting catheter repositioning in our experiments. In this case, the false positive rate was $5.8 \%$. We decided on the VE for displacement detection, because it turned out to be a much more stable reference than the MPE. This can be seen, e.g., by taking a look at their mean errors and maximum differences, see Fig. 3(b).

\section{Discussion and Conclusions}

The results indicate that our catheter localization and tracking algorithms are accurate enough to meet clinical needs, cf. Fig. 3(a). In our experiments, involving non-ECG-triggered X-ray data acquired under free breathing conditions, only tracking of the CFM was accurate enough to be directly applicable to motion compensation without any need for a more sophisticated motion model, see Fig. 3(b), The tracking of the circumferential mapping catheter yielded an 


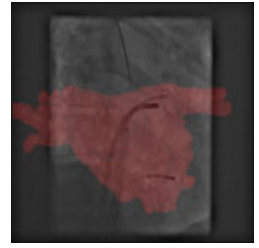

(a)

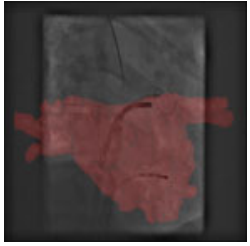

(b)

Fig. 4. (a) Motion compensation using the circumferential mapping catheter. (c) Motion compensation using the most proximal electrode on the coronary sinus catheter.

overall average error of $0.55 \mathrm{~mm}$. Since this error also contains some model error of the underlying B-spline catheter model, which is not adapted over time, the actual tracking performance of the distance-transform-based method is even better. The motion difference between the real motion at the PV ostia and the estimated motion, yielded a maximum error of $2.06 \mathrm{~mm}$. The same error for the MPE was $11.80 \mathrm{~mm}$ and $7.14 \mathrm{~mm}$ for the VE, respectively, see Fig. 3(b). From these numbers, we conclude that the circumferential mapping catheter is the best surrogate for the motion of the left atrium. A visual comparison between motion compensation using the CFM and the VE is given in Fig. 4 At first sight, our observations seem to contradict the results reported in [6]. Maybe the varying results are due to differences in how the procedures were performed. For example, some centers apply general anesthesia while only mild sedation was used in our cases. Some clinical sites also provide a setup where ECG signals can be recorded on the fluoroscopy system. The ECG could be exploited to select proper fluoroscopic frames. As our cases came from multiple sites using different ECG recording equipment, we decided to not take advantage of any ECG signals to keep things consistent. The choice for one method or the other may come down to how well you control the procedure. For example, if there is general anesthesia, stable sinus rhythm, and available ECG information, the approach presented in [6] may be the method of choice. However, in the general case it may not be straightforward to apply it as successfully. Although we found it difficult to rely on the CS catheter for motion compensation, we observed that it could be used to detect displacement of the CFM catheter. If the distance between the circumferential mapping catheter and the virtual electrode changes by a certain amount, we assume that the mapping catheter has been moved from one PV to the other. From our experiments, using the absolute distance between the CFM and the VE yielded the best results to detect that the CFM moved away from a particular PV. A change in the absolute distance of $5 \%$ was the best threshold in our experiments yielding a false positive rate of $5.8 \%$. Compared to a misdetection which may lead to incorrect fluoroscopic overlays, a false detection is preferred. At most, there are a few frames without motion correction. As the catheter displacement approach has only been evaluated on five sequences so far, further validation on a more comprehensive data set is currently underway. 


\section{References}

1. Gage, B., Waterman, A., Shannon, W., Boechler, M., Rich, M., Radford, M.: Validation of Clinical Classification Schemes for Predicting Stroke. Journal of the American Medical Association 285(22), 2864-2870 (2001)

2. Calkins, H., Brugada, J., Packer, D., Cappato, R., Chen, S., Crijns, H., Damiano, R., Davies, D., Haines, D., Haïssaguerre, M., Iesaka, Y., Jackman, W., Jais, P., Kottkamp, H., Kuck, K., Lindsay, B., Marchlinski, F., McCarthy, P., Mont, J., Moradi, F., Nademanee, K., Natale, A., Pappone, C., Prystowsky, E., Raviele, A., Ruskin, J., Shemin, R.: HRS/EHRA/ECAS Expert Consensus Statement on Catheter and Surgical Ablation of Atrial Fibrillation: Recommendations for Personnel, Policy, Procedures and Follow-Up. Heart Rhythm 4(6), 1-46 (2007)

3. Prümmer, M., Hornegger, J.: Cardiac C-arm CT: a unified framework for motion estimation and dynamic CT. IEEE Transactions on Medical Imaging 28(11), 1836 1849 (2009)

4. Ector, J., De Buck, S., Huybrechts, W., Nuyens, D., Dymarkowski, S., Bogaert, J., Maes, F., Heidbüchel, H.: Biplane three-dimensional augmented fluoroscopy as single navigation tool for ablation of atrial fibrillation: Accuracy and clinical value. Heart Rhythm 5(7), 957-964 (2008)

5. Brost, A., Liao, R., Hornegger, J., Strobel, N.: 3-D respiratory motion compensation during EP procedures by image-based 3-D lasso catheter model generation and tracking. In: Yang, G.-Z., Hawkes, D., Rueckert, D., Noble, A., Taylor, C. (eds.) MICCAI 2009. LNCS, vol. 5761, pp. 394-401. Springer, Heidelberg (2009)

6. Ma, Y., King, A.P., Gogin, N., Rinaldi, C.A., Gill, J., Razavi, R., Rhode, K.S.: Real-time respiratory motion correction for cardiac electrophysiology procedures using image-based coronary sinus catheter tracking. In: Jiang, T., Navab, N., Pluim, J.P.W., Viergever, M.A. (eds.) MICCAI 2010. LNCS, vol. 6361, pp. 391399. Springer, Heidelberg (2010)

7. Brost, A., Liao, R., Hornegger, J., Strobel, N.: Model-based registration for motion compensation during EP ablation procedures. In: Fischer, B., Dawant, B.M., Lorenz, C. (eds.) WBIR 2010. LNCS, vol. 6204, pp. 234-245. Springer, Heidelberg (2010)

8. Brost, A., Wimmer, A., Liao, R., Hornegger, J., Strobel, N.: Catheter tracking: Filter-based vs. Learning-based. In: Goesele, M., Roth, S., Kuijper, A., Schiele, B., Schindler, K. (eds.) Pattern Recognition. LNCS, vol. 6376, pp. 293-302. Springer, Heidelberg (2010)

9. Breiman, L., Friedman, J., Olshen, R., Stone, C.: Classification and Regression Trees. Chapman \& Hall, New York (1984)

10. Freund, Y., Schapire, R.: A decision-theoretic generalization of on-line learning and an application to boosting. Journal of Computer and System Sciences 55(1), 119-139 (1997)

11. Breu, H., Gil, J., Kirkpatrick, D., Werman, M.: Linear time Euclidean distance transform algorithms. IEEE Transactions on Pattern Analysis and Machine Intelligence 17(5), 529-533 (1995)

12. Wu, W., Chen, T., Barbu, A., Wang, P., Strobel, N., Zhou, S., Comaniciu, D.: Learning-based Hypothesis Fusion for Robust Catheter Tracking in 2D X-ray Fluoroscopy. To Appear IEEE Conference on Computer Vision and Pattern Recognition, CVPR 2011 (2011)

13. Tu, Z.: Probabilistic boosting-tree: Learning discriminative models for classification, recognition, and clustering. In: Proceedings of the Tenth IEEE International Conference on Computer Vision, ICCV 2005, vol. 2, pp. 1589-1596. IEEE Computer Society, Los Alamitos (2005) 